\title{
Simulação da Variação do Espaçamento na Viabilidade Econômica de um Sistema Agroflorestal
}

\author{
Sidney Araujo Cordeiro ${ }^{1}$, Marcio Lopes da Silva ${ }^{2}$, \\ Silvio Nolasco de Oliveira Neto ${ }^{2}$, Tiago Moreira Oliveira ${ }^{1}$ \\ ${ }^{1}$ Universidade Federal do Piauí - UFPI, Bom Jesus/PI, Brasil \\ ${ }^{2}$ Universidade Federal de Viçosa - UFV, Viçosa/MG, Brasil
}

\begin{abstract}
RESUMO
O objetivo deste estudo foi realizar simulação, verificando a rentabilidade de um sistema agrossilvicultural composto por eucalipto, arroz, soja e gado, de acordo com a variação do espaçamento de plantio do eucalipto e dos sistemas agroflorestais, bem como comparar os resultados com o plantio convencional do eucalipto. A análise econômica foi realizada mediante os métodos de avaliação de projetos florestais, Valor Presente Líquido (VPL), Benefício Periódico Equivalente (BPE), Razão Benefício/Custo (B/C) e Taxa Interna de Retorno (TIR). Todos os projetos analisados apresentaram-se viáveis economicamente, sendo que o eucalipto em monocultivo apresentou melhores resultados. Verificou-se que o ganho em área com plantio das culturas agrícolas e criação de gado não obtém o mesmo retorno econômico caso a área estivesse com plantio de árvores.
\end{abstract}

Palavras-chave: agrossilvicultura, eucalipto, rentabilidade.

\section{Influence of Spacing Simulation in the Economic Viability of an Agroforestry System}

\begin{abstract}
This study aimed to simulate and verify the rentability of an agroforestry system composed of eucalipt, rice, soybean e cattle, according to the spacing variation in the plantation of eucalypt and agroforestry system, and compare the results with conventional eucalypt planting. Financial analysis was performed through the forestry project evaluation methods, Net Present Value (VPL), Equivalent Annual Value (VAE), Benefit/Cost Relation (B/C) and Internal Return Rate (TIR). All projects were shown to be economically viable, with eucalyptus monoculture showing the best results. We verified that the gain in area with planting of crops agricultural and cattle creation did not lead to the same financial return if this area was planted with trees.
\end{abstract}

Keywords: agroforestry system, eucalypt, rentability.

\section{INTRODUÇÃO}

Os sistemas agroflorestais (SAF) vêm despertando atualmente $\mathrm{o}$ interesse dos produtores rurais em razão, principalmente, dos altos custos envolvidos na implantação e manutenção de florestas e devido à necessidade de implementação de projetos que busquem conciliar desenvolvimento econômico e redução de impactos ao meio ambiente. De acordo com Souza et al. (2007), a prática desse tipo específico de sistema produtivo 
diversifica a produção agropecuária em uma propriedade rural, podendo envolver uma variabilidade de espécies tanto agrícolas quanto florestais e mesmo pecuárias num só sistema, com vistas à maximização do lucro.

O espaçamento é um dos fatores que mais sofre variação nas diferentes modalidades de sistemas agroflorestais, sendo importante nos estudos de análise econômica e de simulação deles (Cordeiro, 2010). Dependendo do SAF, o espaçamento pode ser responsável por resultados negativos ou positivos nos fluxos de caixa do investimento (Burner \& Brauer, 2003).

A experiência a respeito de espaçamento de plantio de florestas no Brasil tem-se limitado às florestas de produção, normalmente plantios equiâneos puros, que têm como objetivos principais a quantidade e a qualidade da madeira produzida. Vários autores, dentre eles Silva et al. (1995), Oliveira et al. $(2003,2010)$ e Rondon (2006), por exemplo, apresentam análises de estudos de espaçamentos para florestas de produção.

A atividade agroflorestal reúne em seu processo produtivo uma série de etapas decorrentes das práticas agrícolas, pecuárias e florestais necessárias à condução e ao manejo das espécies que compõem esses sistemas. Por esse motivo, a análise financeira de um cenário agroflorestal se torna complexa, uma vez que envolve a combinação de diversas variáveis técnicas e custos, informações muitas vezes não facilmente disponíveis (Bentes-Gama et al., 2005). A análise econômica de sistemas agroflorestais é de grande importância para o produtor rural, propiciando um melhor conhecimento dos custos e receitas da atividade (Varela \& Santana, 2009).

Com relação à análise da viabilidade econômica em SAF, podem-se citar alguns trabalhos, dentre eles os de Bentes-Gama et al. (2005), Dubé et al. (2002) e Rodrigues et al. (2007). Os estudos relacionando espaçamento e viabilidade econômica em SAF são escassos.

Diante do exposto, este trabalho busca contribuir para a diminuição da carência de pesquisas nessa área, tendo como objetivo verificar a rentabilidade por meio de simulação de um sistema agrossilvicultural composto por eucalipto, arroz, soja e gado, de acordo com a variação do espaçamento de plantio do eucalipto e dos sistemas agroflorestais, bem como comparar os resultados com os do plantio convencional do eucalipto.

\section{MATERIAL E MÉTODOS}

\subsection{Custos e receitas da floresta de eucalipto em plantio convencional}

Considerou-se o plantio do eucalipto no espaçamento de $3 \times 3 \mathrm{~m}$, totalizando 1.111 árvores por hectare, com o objetivo de converter $40 \%$ da madeira em carvão no sétimo ano e o restante visando à produção de madeira serrada aos 14 anos da floresta.

Para o cálculo do volume de madeira, levou-se em consideração que a floresta tem um Incremento Médio Anual (IMA) de $35 \mathrm{~m}^{3} /$ ha, com uma produtividade esperada de $245 \mathrm{~m}^{3}$ /ha, no $7^{\circ}$ ano, e de $295 \mathrm{~m}^{3}$ /ha no $14^{\circ}$ ano. Considerou-se um fator de conversão volumétrica de lenha para carvão $\left(\mathrm{m}^{3} / \mathrm{mdc}\right)$ de $1,5: 1$, obtendo-se 97,33 metros de carvão (mdc) aos 7 anos. Os custos são apresentados na Tabela 1 .

O custo de carbonização foi calculado com base em dados de Cordeiro (2010), sendo o custo de produção de $\mathrm{R} \$ 30,00 / \mathrm{mdc}$

Tabela 1. Custos do projeto florestal visando a produção de carvão e madeira para serraria.

Table 1. Costs common to reforestation project for charcoal and wood production.

\begin{tabular}{|c|c|c|}
\hline Atividade & Ano de ocorrência & Custos (R\$/ha) \\
\hline Implantação & 1 & $2.500,00$ \\
\hline Manutenção & 2 & 728,98 \\
\hline Manutenção & 3 & 538,01 \\
\hline Manutenção & 8 & 666,86 \\
\hline Manutenção & 9 & 464,04 \\
\hline Manutenção & 4 a 6 e 10 a 13 & 495,89 \\
\hline Colheita e carbonização & 7 & $5.880,00$ \\
\hline Colheita e processamento & 14 & $19.889,10$ \\
\hline
\end{tabular}

Fonte: Cordeiro (2010) e empresas do setor. 
O custo total da serraria por metro cúbico de madeira serrada foi obtido de Souza et al. (2007), sendo de $\mathrm{R} \$ 67,65$, composto pelos seguintes itens: mão de obra (salário + encargos $=\mathrm{R} \$ 51,01$ ), materiais e peças $(R \$ 2,86)$, energia elétrica $(R \$ 7,92)$ e depreciação ( $\$$ \$ 5,86). Não foram incluídos os custos relacionados ao pagamento de taxas e impostos (ICMS), ou seja, o custo $/ \mathrm{m}^{3}$ de madeira serrada se refere apenas aos gastos para serrar a madeira e estocá-la no pátio da indústria.

Os preços de venda do carvão e da madeira serrada foram obtidos de acordo com empresas privadas e produtores rurais da região, sendo de $\mathrm{R} \$ 112,50$ e $\mathrm{R} \$ 300,00$, respectivamente.

\subsection{Custos e receitas do sistema agrossilvicultural}

\subsubsection{SAF com eucalipto no espaçamento $10 \times 4 m$ (250 árvores/ha)}

O sistema agrossilvipastoril apresentado aqui consiste em plantar o eucalipto no espaçamento amplo $(10 \times 4 \mathrm{~m})$, ou seja, 250 árvores por hectare, juntamente com o arroz nas entrelinhas. Após a colheita do arroz, planta-se a soja. Após a colheita da soja, planta-se a Brachiaria. Após a formação da pastagem introduz-se o novilho com aproximadamente 5 arrobas. Após 2 anos vende-se o boi gordo. Portanto, a cada 2 anos uma nova remessa de novilhos é colocada na área.
Considerou-se que a produtividade da floresta é de $248 \mathrm{~m}^{3} /$ há e que $40 \%$ da madeira produzida é para energia e $60 \%$ é para serraria.

Aos 7 anos obtém-se a madeira para a produção de carvão e no final de 14 anos colhe-se a madeira e encerra-se esse ciclo do projeto. A produção e as receitas desse sistema encontram-se na Tabela 2.

\subsubsection{SAF com eucalipto no espaçamento $10 \times 6 \mathrm{~m}$ (166 árvores/ha)}

O volume estimado foi de $164,67 \mathrm{~m}^{3} / \mathrm{ha}$, sendo que $40 \%$ da madeira produzida é para energia e $60 \%$ é para serraria.

Aos 7 anos obtém-se a madeira para a produção de carvão e no final de 14 anos colhe-se a madeira e encerra-se esse ciclo do projeto.

A produção e receitas para esse sistema encontram-se na Tabela 3.

\subsubsection{SAF com eucalipto no espaçamento $10 \times 8 \mathrm{~m}$ (125 árvores/ha)}

O volume estimado foi de $124 \mathrm{~m}^{3} / \mathrm{ha}$, sendo que $40 \%$ da madeira produzida é para energia e $60 \%$ é para serraria.

Aos 7 anos obtém-se a madeira para a produção de carvão e no final de 14 anos colhe-se a madeira e encerra-se esse ciclo do projeto.

Tabela 2. Produção e receita do sistema agrossilvipastoril.

Table 2. Production and income to agroforestry system.

\begin{tabular}{lcccc}
\multicolumn{1}{c}{ Discriminação do produto } & Unidade (un) & $\begin{array}{c}\text { Produção } \\
\text { (un/ha) }\end{array}$ & $\begin{array}{c}\text { Preço de venda } \\
\text { (R\$/un) }\end{array}$ & Receita (R\$/ha) \\
\hline - Arroz & Sc & 23,33 & 26,5 & 618,25 \\
- Soja & Sc & 25 & 43,5 & $1.087,50$ \\
- Boi gordo & @ & 15 & 71 & $1.065,00$ \\
- Madeira de eucalipto para serraria & $\mathrm{m}^{3}$ & 148 & 570 & $84.360,00$ \\
\hline - Madeira de eucalipto para energia & $\mathrm{mdc}$ & 50 & 112,5 & $5.625,00$ \\
\hline
\end{tabular}

Tabela 3. Produção e receita do sistema agrossilvipastoril.

Table 3. Production and income to agroforestry system.

\begin{tabular}{lcccc}
\multicolumn{1}{c}{ Discriminação do produto } & Unidade (un) & $\begin{array}{c}\text { Produção } \\
\text { (un/ha) }\end{array}$ & $\begin{array}{c}\text { Preço de venda } \\
\text { (R\$/un) }\end{array}$ & Receita (R\$/ha) \\
\hline - Arroz & Sc & 23,526 & 26,5 & 623,44 \\
- Soja & Sc & 25,21 & 43,5 & $1.096,63$ \\
\hline - Boi gordo & $@$ & 15,126 & 71 & $1.073,95$ \\
- Madeira de eucalipto para serraria & $\mathrm{m}^{3}$ & 98,8 & 570 & $56.316,00$ \\
\hline - Madeira de eucalipto para energia & $\mathrm{mdc}$ & 32,94 & 112,5 & $3.705,75$ \\
\hline
\end{tabular}


A produção e receitas para esse sistema se encontram na Tabela 4.

\subsection{Análise econômica}

A análise financeira de todos os sistemas foi embasada nos métodos de avaliação de projetos apresentados a seguir.

\subsubsection{Valor Presente Líquido - VPL}

A viabilidade econômica de um projeto analisada pelo VPL é indicada pela diferença positiva entre receitas e custos atualizados para uma determinada taxa de desconto (Rezende \& Oliveira, 2008; Silva et al., 2005), conforme Equação 1. O critério de adoção deste método é o seguinte: um VPL positivo indica que o projeto é economicamente viável para uma determinada taxa utilizada.

$V P L=\sum_{j-1}^{n} \frac{R_{j}}{(1+i)^{j}}-\sum_{j-1}^{n} \frac{C_{j}}{(1+i)^{j}}$

em que: $R_{j}=$ receitas no período $j ; C_{j}=$ custos no período $j ; i=$ taxa de desconto; $j=$ período de ocorrência de $R_{j}$ e $C_{j}$; e $n=$ duração do projeto, em anos, ou em número de períodos de tempo.

\subsubsection{Benefício Periódico Equivalente - BPE}

O Benefício Periódico Equivalente (BPE) é a parcela periódica e constante necessária ao pagamento de uma quantia igual ao VPL da opção de investimento em análise ao longo de sua vida útil, conforme Equação 2. O projeto será considerado economicamente mais atrativo quanto maior for o valor do benefício periódico equivalente (Rezende \& Oliveira, 2008; Silva et al., 2005).

$$
B P E=\frac{V P L \cdot i}{1-(1+i)^{-n}}
$$

em que: $V P L=$ valor presente líquido; e $n=$ duração do ciclo ou rotação em anos.

\subsubsection{Razão Benefício/Custo - B/C}

Este método consiste em determinar a relação entre o valor presente dos benefícios e o valor presente dos custos para uma determinada taxa de juros ou descontos (Silva et al., 2005), conforme Equação 3. Um projeto é considerado viável economicamente se $B / C>1$. Entre dois ou mais projetos, o mais viável é aquele que apresentar o maior valor de $B / C$ (Rezende \& Oliveira, 2008). Quando $B / C=1$, resulta em $V P L=0$.

$B / C=\frac{\sum_{j=0}^{n} R_{j}(1+i)^{-j}}{\sum_{j=0}^{n} C_{j}(1+i)^{-j}}$

em que: $R_{j}=$ receita no final do ano $j ; C_{i}=$ custo no final do ano $j$; e $n=$ duração do projeto, em anos.

\subsubsection{Taxa Interna de Retorno - TIR}

A TIR é a taxa de desconto que iguala o valor atual das receitas futuras ao valor atual dos custos do projeto, constituindo uma medida relativa que reflete o aumento no valor do investimento ao longo do tempo, com base nos recursos requeridos para produzir o fluxo de receitas (Rezende \& Oliveira, 2008; Silva et al., 2005), conforme Equação 4.

$$
\sum_{j=1}^{n} \frac{R_{j}}{(1+T I R)^{j}}-\sum_{j=1}^{n} \frac{C_{j}}{(1+T I R)^{j}}=0
$$

em que: $T I R=$ taxa interna de retorno; as demais variáveis já foram definidas.

Utilizou-se na análise a taxa de juros de $8,75 \%$ ao ano. Essa taxa de juros é empregada, normalmente, pelo Programa de Plantio Comercial de Florestas (PROPFLORA) para produtores (pessoas físicas e

Tabela 4. Produção e receita do sistema agrossilvipastoril.

Table 4. Production and income to agroforestry system.

\begin{tabular}{lcccc}
\multicolumn{1}{c}{ Discriminação do produto } & Unidade (un) & $\begin{array}{c}\text { Produção } \\
\text { (un/ha) }\end{array}$ & $\begin{array}{c}\text { Preço de venda } \\
\text { (R\$/un) }\end{array}$ & Receita (R\$/ha) \\
\hline - Arroz & Sc & 23,62 & 26,5 & 625,93 \\
- Soja & Sc & 25,32 & 43,5 & $1.101,42$ \\
\hline - Boi gordo & @ & 15,19 & 71 & $1.078,49$ \\
- Madeira de eucalipto para serraria & $\mathrm{m}^{3}$ & 74,4 & 570 & $42.408,00$ \\
\hline - Madeira de eucalipto para energia & $\mathrm{mdc}$ & 24,8 & 112,5 & $2.790,00$ \\
\hline
\end{tabular}


jurídicas), com escopo de implantação e manutenção das florestas para uso comercial, energético e processamento industrial.

\section{RESULTADOS E DISCUSSÃO}

\subsection{Eucalipto em plantio convencional $(3 \times 3 \mathrm{~m})$}

O fluxo de caixa para o projeto está apresentado na Tabela 5, sendo construído anualmente, visando um horizonte de planejamento de 14 anos. As receitas são obtidas nos anos 7 e 14 .

O Valor Presente Líquido (VPL) do projeto analisado foi maior do que zero, significando que é viável economicamente, sendo de R \$48.996,50/ha. Vale lembrar que o VPL representa o lucro do negócio corrigido pela taxa de juros $(8,75 \%$ a. a.), para o horizonte de 14 anos.

A Taxa Interna de Retorno (TIR) é a taxa que representa o retorno financeiro do projeto. A TIR obtida foi de $29 \%$ e, portanto, foi maior do que a taxa de desconto, que, no caso, é de $8,75 \%$ ao ano, o que significa que o projeto é viável, sob esse ponto de vista.

O projeto é viável, uma vez que o Benefício Periódico Equivalente (BPE) se apresentou positivo, e que representa o lucro anual do negócio. De acordo com os resultados, o lucro anual foi de $\mathrm{R} \$ 6.204,53 /$ ha.
A Razão Benefício/Custo foi maior que 1, indicando também que o projeto é viável economicamente. Essa razão foi de 4,26, o que significa que as receitas superam os custos em quatro vezes.

Resultados semelhantes foram encontrados por Contreras Marquez (1997). O autor analisou a viabilidade econômica de plantios de Eucalyptus camaldulensis e Eucalyptus pellita, com 7 anos de idade, estabelecidos nos espaçamentos $9 \times 9 \mathrm{~m}, 6 \times 4 \mathrm{~m}, 3 \times 6 \mathrm{~m}, 3 \times 5 \mathrm{~m}$, $3 \times 4 \mathrm{~m}, 3 \times 3 \mathrm{~m}, 3 \times 2 \mathrm{~m}, 3 \times 1,5 \mathrm{~m}$ e $3 \times 1 \mathrm{~m}$ na região de cerrado de Minas Gerais. Os povoamentos nesse espaçamento $3 \times 3 \mathrm{~m}$ apresentaram Taxa Interna de Retorno (TIR) de 11,72\% e Valores Presente Líquido (VPL) e Esperado da Terra (VET) de US\$ 596,00 e US $\$ 844,00 /$ ha, respectivamente. Para E. camaldulensis, a maior produção foi obtida no espaçamento de $3 \times 2 \mathrm{~m}$ (199 st/ha), não obstante o povoamento no espaçamento de $3 \mathrm{x}$ ter sido o mais viável economicamente, com maior taxa interna de retorno (14,6\%), custo de produção de US\$ 8,24/st e valores presente líquido e esperado da terra de US\$ 974/ha e US\$1.379/ha, respectivamente.

Outro fator a ser levado em consideração é o risco da atividade. Varela \& Santana (2009), ao analisarem o risco envolvido em um sistema agroflorestal e o cultivo de espécies agrícolas em sistema tradicional, verificaram que a função de risco estimada apontou que os SAF apresentaram menor risco que os sistemas tradicionais, evidenciando-se que a aplicação de insumos era fonte

Tabela 5. Fluxo de caixa para o projeto de reflorestamento com eucalipto visando a produção de carvão e madeira para serraria.

Table 5. Cash flow for reforestation project with eucaliptus, for charcoal and wood productio.

\begin{tabular}{ccrcrc}
\hline Ano & Receita & Custo & Receita descontada & Custo descontado & Saldo \\
\hline 1 & - & $2.500,00$ & - & $2.298,85$ & $-2.298,85$ \\
\hline 2 & - & 728,98 & - & 616,39 & $-616,39$ \\
\hline 3 & - & 538,01 & - & 418,31 & $-418,31$ \\
\hline 4 & - & 495,89 & - & 354,54 & $-354,54$ \\
\hline 5 & - & 495,89 & - & 326,02 & $-326,02$ \\
\hline 6 & - & 495,89 & - & 299,78 & $-299,78$ \\
\hline 7 & $22.050,00$ & $5.880,00$ & $12.257,55$ & $3.268,68$ & $8.988,87$ \\
\hline 8 & - & 666,86 & - & 340,88 & $-340,88$ \\
\hline 9 & - & 464,04 & - & 218,12 & $-218,12$ \\
\hline 10 & - & 495,89 & - & 214,33 & $-214,33$ \\
\hline 11 & - & 495,89 & - & 197,09 & $-197,09$ \\
\hline 12 & - & 495,89 & - & 181,23 & $-181,23$ \\
\hline 13 & - & 495,89 & - & 166,65 & $-166,65$ \\
\hline 14 & $167.580,00$ & $19.889,10$ & $51.786,01$ & $6.146,18$ & $45.639,83$ \\
\hline Total & $189.630,00$ & $34.138,22$ & $64.043,56$ & $15.047,07$ & $48.996,50$ \\
\hline
\end{tabular}


de redução de risco, mas a tecnologia adotada precisa ser adequada, pois se apresenta como fator de aumento de risco nos dois sistemas.

\subsection{SAF com eucalipto no espaçamento $10 \times 4 m$}

Os resultados financeiros para o sistema agroflorestal analisado no espaçamento $10 \times 4 \mathrm{~m}$ encontram-se na Tabela 6.

O Valor Presente Líquido (VPL) do projeto analisado foi maior do que zero, significando que é viável economicamente, sendo de $\mathrm{R} \$ 23.005,37 /$ ha.

A Taxa Interna de Retorno (TIR) foi de $25 \%$. A TIR obtida foi maior do que a taxa de desconto que, no caso, é de $8,75 \%$ ao ano, o que significa que o projeto é viável, sob esse ponto de vista.

O projeto é viável, uma vez que o Benefício Periódico Equivalente (BPE), que representa o lucro anual do negócio, foi positivo, sendo de R $2.913,22 /$ ha.

A Razão Benefício/Custo foi de 3,13, indicando, também, que o projeto é viável economicamente.

Souza et al. (2007) analisaram a viabilidade econômica de talhões de um clone de híbridos naturais de Eucalyptus urophylla $\times$ Eucalyptus camaldulensis plantado em consórcio com arroz, soja e pastagem no espaçamento 10 x 4 metros. Concluíram que houve um aumento significativo na viabilidade econômica do sistema agroflorestal, à medida que se agregou valor aos produtos florestais. A viabilidade econômica do sistema depende mais da atividade florestal e da pecuária do que das atividades anuais.

\subsection{SAF com eucalipto no espaçamento $10 \times 6 \mathrm{~m}$}

Na Tabela 7 são apresentados os valores de receitas e custos para o sistema agrossilvicultural com eucalipto no espaçamento $10 \times 6 \mathrm{~m}$. Observa-se que a partir do quarto ano as receitas começam a superar os custos envolvidos no projeto.

O Valor Presente Líquido (VPL) do projeto analisado foi maior do que zero, significando que é viável economicamente, sendo de R\$12.979,14/ha.

A Taxa Interna de Retorno (TIR) foi de $22 \%$, significando que o projeto é viável.

O projeto é viável, uma vez que o Benefício Periódico Equivalente (BPE), que representa o lucro anual do negócio, foi positivo, sendo de R\$1.643,58/ha.

$A$ razão $B / C$ foi maior que 1 , indicando também que o projeto é viável economicamente, sendo de 2,76.

\subsection{SAF com eucalipto no espaçamento $10 \times 8 \mathrm{~m}$}

Na Tabela 8 apresenta-se o fluxo de caixa para o sistema agroflorestal com eucalipto plantado no espaçamento $10 \times 8 \mathrm{~m}$, consorciado com arroz, soja e boi gordo.

Tabela 6. Fluxo de caixa para o sistema agrossilvicultural.

Table 6. Cash flow for agroforestry system.

\begin{tabular}{|c|c|c|c|c|c|}
\hline Ano & Receita & Custo & $\begin{array}{c}\text { Receita } \\
\text { descontada }\end{array}$ & $\begin{array}{c}\text { Custo } \\
\text { descontado }\end{array}$ & Saldo \\
\hline 1 & 618,25 & $1.616,00$ & 568,5 & $1.485,98$ & $-917,47$ \\
\hline 2 & $1.087,50$ & $1.590,49$ & 919,54 & $1.344,85$ & $-425,31$ \\
\hline 3 & - & 816,46 & - & 634,81 & $-634,81$ \\
\hline 4 & $1.065,00$ & $1.061,84$ & 761,43 & 759,18 & 2,26 \\
\hline 5 & - & 293,84 & - & 193,18 & $-193,18$ \\
\hline 6 & 1.065 & 643,84 & 643,83 & 389,23 & 254,61 \\
\hline 7 & 5.625 & $3.000,00$ & $3.126,92$ & $1.667,69$ & $1.459,23$ \\
\hline 8 & 1.065 & 643,84 & 544,39 & 329,11 & 215,28 \\
\hline 9 & - & 293,84 & - & 138,12 & $-138,12$ \\
\hline 10 & 1.065 & 643,84 & 460,31 & 278,28 & 182,03 \\
\hline 11 & - & 293,84 & - & 116,79 & $-116,79$ \\
\hline 12 & 1.065 & 643,84 & 389,22 & 235,3 & 153,92 \\
\hline 13 & - & 293,84 & - & 98,75 & $-98,75$ \\
\hline 14 & 85.425 & $10.147,50$ & $26.398,25$ & $3.135,81$ & $23.262,45$ \\
\hline Total & $98.080,75$ & $21.983,01$ & $33.812,44$ & $10.807,07$ & $23.005,37$ \\
\hline
\end{tabular}


Tabela 7. Fluxo de caixa para o sistema agrossilvicultural.

Table 7. Cash flow for agroforestry system.

\begin{tabular}{|c|c|c|c|c|c|}
\hline Ano & Receita & Custo & $\begin{array}{c}\text { Receita } \\
\text { descontada }\end{array}$ & $\begin{array}{c}\text { Custo } \\
\text { descontado }\end{array}$ & Saldo \\
\hline 1 & 623,44 & $1.616,00$ & 573,27 & $1.485,98$ & $-912,7$ \\
\hline 2 & $1.096,63$ & $1.590,49$ & 927,26 & $1.344,85$ & $-417,59$ \\
\hline 3 & - & 816,46 & - & 634,81 & $-634,81$ \\
\hline 4 & $1.073,95$ & $1.061,84$ & 767,83 & 759,18 & 8,66 \\
\hline 5 & - & 293,84 & - & 193,18 & $-193,18$ \\
\hline 6 & $1.073,95$ & 643,84 & 649,24 & 389,23 & 260,02 \\
\hline 7 & $3.705,75$ & 988,2 & 2060,01 & 549,34 & $1.510,68$ \\
\hline 8 & $1.073,95$ & 643,84 & 548,97 & 329,11 & 219,86 \\
\hline 9 & - & 293,84 & - & 138,12 & $-138,12$ \\
\hline 10 & $1.073,95$ & 643,84 & 464,18 & 278,28 & 185,9 \\
\hline 11 & - & 293,84 & - & 116,79 & $-116,79$ \\
\hline 12 & $1.073,95$ & 643,84 & 392,49 & 235,3 & 157,19 \\
\hline 13 & - & 293,84 & - & 98,75 & $-98,75$ \\
\hline 14 & $56.316,00$ & $6.683,82$ & 17402,91 & $2.065,45$ & $15.337,47$ \\
\hline Total & $67.111,57$ & $16.507,53$ & $23.786,20$ & $8.618,36$ & $15.167,85$ \\
\hline
\end{tabular}

Tabela 8. Fluxo de caixa para o sistema agrossilvicultural.

Table 8. Cash flow for agroforestry system.

\begin{tabular}{cccccc|}
\hline Ano & Receita & Custo & $\begin{array}{c}\text { Receita } \\
\text { descontada }\end{array}$ & $\begin{array}{c}\text { Custo } \\
\text { descontado }\end{array}$ & Saldo \\
\hline 1 & 625,93 & $1.616,00$ & 575,5678161 & $1.485,98$ & $-910,41$ \\
\hline 2 & $1.101,42$ & $1.590,49$ & 931,3103448 & $1.344,85$ & $-413,54$ \\
\hline 3 & - & 816,46 & - & 634,81 & $-634,81$ \\
\hline 4 & $1.078,49$ & $1.061,84$ & 771,0793278 & 759,18 & 11,9 \\
\hline 5 & - & 293,84 & - & 193,18 & $-193,18$ \\
\hline 6 & $1.078,49$ & 643,84 & 651,9893906 & 389,23 & 262,76 \\
\hline 7 & $2.790,00$ & 744 & 1550,955524 & 413,59 & $1.137,37$ \\
\hline 8 & $1.078,49$ & 643,84 & 551,29239 & 329,11 & 222,18 \\
\hline 9 & - & 293,84 & - & 138,12 & $-138,12$ \\
\hline 10 & $1.078,49$ & 643,84 & 466,1476148 & 278,28 & 187,87 \\
\hline 11 & - & 293,84 & - & 116,79 & $-116,79$ \\
\hline 12 & $1.078,49$ & 643,84 & 394,1530895 & 235,3 & 158,85 \\
\hline 13 & - & 293,84 & - & 98,75 & $-98,75$ \\
\hline 14 & $42.408,00$ & $5.033,16$ & 13105,0316 & $1.555,36$ & $11.549,67$ \\
\hline Total & $52.317,80$ & $14.612,67$ & $18.997,53$ & $7.972,52$ & $11.025,01$ \\
\hline
\end{tabular}

O Valor Presente Líquido (VPL) do projeto analisado foi maior do que zero, R\$ 11.025,01/ha, significando que ele é viável economicamente.

A Taxa Interna de Retorno (TIR) foi de 19\%, significando que o projeto é viável.

O projeto é viável uma vez que o Benefício Periódico Equivalente (BPE) se apresentou positivo, de R $\$ 1.396,12$, o que representa o lucro anual do negócio.

A Razão Benefício/Custo foi de 2,4, maior que 1, indicando também que o projeto é viável economicamente.

\section{CONCLUSÕES}

- Todos os projetos analisados apresentaram-se viáveis economicamente, de acordo com os critérios de análise econômica utilizados, sendo que, nesta pesquisa, o eucalipto em monocultivo foi o projeto mais rentável, que apresentou melhores resultados. O cultivo de árvores para carvão e serraria nos sistemas agrossilvipastoris analisados mostrou-se um investimento altamente rentável. 
- Na medida em que se aumenta o espaçamento de plantio das árvores de eucalipto, tem-se um aumento da área disponível para plantio de arroz, soja e criação de gado. Mas, de acordo com os resultados deste trabalho, esse ganho em área não obtém o mesmo retorno financeiro se essa área for destinada ao plantio de árvores, considerando-se as condições analisadas no trabalho.

\section{STATUS DA SUBMISSÃO}

Recebido: 15 mar., 2013

Aceito: 12 mar., 2017

\section{AUTOR(ES) PARA CORRESPONDÊNCIA}

\section{Sidney Araujo Cordeiro}

Departamento de Engenharias, Universidade Federal do Piauí - UFPI, Rodovia Municipal Bom Jesus, Viana, Km 1, Planalto Horizonte, CEP 64900-000, Bom Jesus, PI, Brasil e-mail: sidney.cordeiro@ufvjm.edu.br

\section{REFERENNCIAS}

Bentes-Gama MM, Silva ML, Vilcahuamán LJM, Locatelli M. Análise econômica de sistemas agroflorestais na Amazônia Ocidental, Machadinho D'Oeste - RO. Revista Árvore 2005; 29(3): 401-411. http://dx.doi.org/10.1590/ S0100-67622005000300007.

Burner DM, Brauer DK. Herbage response to spacing of loblolly pine trees in a minimal management silvopasture in southeastern USA. Agroforestry Systems 2003; 57(1): 69-77. http://dx.doi.org/10.1023/A:1022943224478.

Contreras Marquez CE. Estudo silvicultural e econômico de povoamentos de eucalipto na região de cerrado de Minas Gerais [tese]. Viçosa: Universidade Federal de Viçosa; 1997.

Cordeiro SA. Avaliação econômica e simulação em sistemas agroflorestais [tese]. Viçosa: Universidade Federal de Viçosa; 2010.
Dubé F, Couto L, Silva ML, Leite HG, Garcia R, Araujo GAA. A simulation model for evaluating technical and economic aspects of an industrial eucalyptus-based agroforestry system in Minas Gerais, Brazil. Agroforestry Systems 2002; 55(1): 73-80. http://dx.doi.org/10.1023/A:1020240107370.

Oliveira SN No, Reis GG, Reis MGF, Leite HG, Neves JCL. Crescimento e distribuição diamétrica de Eucalyptus camaldulensis em diferentes espaçamentos e níveis de adubação na região de cerrado de Minas Gerais. Revista Floresta 2010; 40(4): 755-762.

Oliveira SN No, Reis GG, Reis MGF, Neves JCL. Produção e distribuição de biomassa em Eucalyptus camaldulensis Dehn. em resposta à adubação e ao espaçamento. Revista Árvore 2003; 27(1): 15-23. http://dx.doi.org/10.1590/ S0100-67622003000100003.

Rezende JLP, Oliveira AD. Análise econômica e social de projetos florestais. Viçosa: UFV; 2008. 386 p.

Rodrigues ER, Cullen L Jr, Beltrame TP, Moscogliato AV, Silva IC. Avaliação econômica de sistemas agroflorestais implantados para recuperação de reserva legal no Pontal do Paranapanema, São Paulo. Revista Árvore 2007; 31(5): 941948. http://dx.doi.org/10.1590/S0100-67622007000500018.

Rondon EV. Estudo de biomassa de Tectona grandis L. f. sob diferentes espaçamentos no Estado de Mato Grosso. Revista Árvore 2006; 30(3): 337-341. http://dx.doi. org/10.1590/S0100-67622006000300003.

Silva ML, Jacovine LAG, Valverde SL. Economia florestal. Viçosa: UFV; 2005.

Silva ML, Machado CC, Ladeira HP. Influência do custo de corte do diâmetro da árvore e do volume por hectare na rotação econômica de povoamentos de eucalipto. Revista Árvore 1995; 19(4): 505-516.

Souza AN, Oliveira AD, Scolforo JRS, Rezende JLP, Mello JM. Viabilidade econômica de um sistema agroflorestal. Revista Cerne 2007; 13(1): 96-106.

Varela LB, Santana AC. Aspectos econômicos da produção e do risco nos sistemas agroflorestais e nos sistemas tradicionais de produção agrícola em Tomé-açu, Pará 2001 a 2003. Revista Árvore 2009; 33(1): 151-160. http:// dx.doi.org/10.1590/S0100-67622009000100016. 